\title{
TOPOLOGY OPTIMIZATION OF PERIODIC STRUCTURES FOR COUPLED ACOUSTIC-STRUCTURE SYSTEMS
}

\author{
William Martins Vicente ${ }^{1}$, Renato Picelli ${ }^{1}$, Renato Pavanello ${ }^{1}$, Yi Min Xie ${ }^{2}$ \\ ${ }^{1}$ Department of Computational Mechanics, Faculty of Mechanical Engineering \\ University of Campinas \\ Rua Mendeleyev 200, 13083-860 Campinas, Brazil \\ vicente@fem.unicamp.br; picelli@fem.unicamp.br; pava@fem.unicamp.br \\ ${ }^{2}$ Centre for Innovative Structures and Materials, School of Engineering \\ RMIT University \\ GPO Box 2476, Melbourne 3001, Australia \\ mike.xie@rmit.edu.au
}

Keywords: Topology optimization, Fluid-structure interaction, Frequency response, Periodic structures.

\begin{abstract}
This work applies the topology optimization technique to an acoustic-structure coupled system with periodic geometry constraint in order to obtain the optimal layout of the design domain for the minimization of the pressure frequency response in the acoustic fluid. The displacement-pressure formulation $(u-p)$ is used for the finite element analysis of the coupled system and external harmonic excitations are applied in the system. The design domain of the coupled system is considered to be composed of identical unit cells. A periodic geometry constraint is applied in the design domain considering the fluid-structure interaction and the objective function. Appling the modified bi-directional evolutionary structural optimization (BESO) technique to the system, the design domain is evolving towards the optimal topology of the unit cells through removing/adding material accordingly to the sensitivity analysis. The influence of the total number of unit cells composing the periodic structure and the aspect ratio of the unit cells are investigated in the minimization of the objective function. In order to show the capability and efficiency of the proposed formulation, two acoustic-structure systems are optimized for several cell configurations, different aspect ratios of the periodic unit cells and excitation frequencies.
\end{abstract}




\section{INTRODUCTION}

In recent years, the optimization of multiphysics systems has been considered in several areas of the computational engineering. Concerning the acoustic-structure systems, the minimization of the pressure frequency response in a region of the fluid domain could be particularly important for engineering problems like noise reduction in interior of rooms, in passenger compartments of automobile, airplane, etc. Different topology optimization approaches, $[1,2,3,4,5,6,7,8]$, have been developed in the last decades and can be used to obtain a better performance of multiphysics systems.

The frequency response problems in multiphysics system have been investigated using different approaches for circumvent the difficulty of optimize systems having more than one material domain $[9,10,11,12]$.

Extending the traditional bi-directional evolutionary structural optimization (BESO) method to a three phases system (structure, fluid end void) and using the displacement-pressure formulation $(u-p)$ for the finite element analysis of the coupled system, the problem concerning the interface between the domains during the optimization process have been studied [13, $14,15]$. Due the characteristics of the $u-p$ formulation and the evolutionary optimization technique, the coupled system during the optimization procedure does not present intermediate densities of structure, thus, all phases, solid, void and fluid, as well as the interface between the domains are explicitly defined.

Concerning the optimization involving periodic structures, the topology optimization has been applied to find optimal design of the periodic cells using the based density approach, [16, 17] and more recently with the evolutionary technique, $[18,19,20]$, considering static and dynamic problems.

Recently, the topology optimization using BESO technique was applied to minimize the frequency response considering the pressure [21] and displacement [22] for a range of frequency of excitation.

This work uses the modified BESO to fluid-structure coupled system to optimize acousticstructure problems where the design domain is subject to a periodic constraint. The analysis of the system is carried out using the displacement-pressure formulation $(u-p)$, where the solid domain is governed by the elasticity equation and the acoustic fluid domain by Helmholtz equation. The surface-coupling integral are used to fully coupled the domains.

This paper is organized as follows: Section 2 presents the acoustic-structure formulation used and the finite element modeling of the coupled system. In Section 3, the topology optimization problem for the pressure frequency response minimization is developed and the sensitivity analysis is carried out considering the periodicity of the unit cells in the design domain. Section 4 shows some numerical results reached using the proposed methodology. Finally, conclusions are drawn in Section 5.

\section{ACOUSTIC-STRUCTURE INTERACTION}

The fluid-structure interaction considered in this work is represented generically in Fig. 1, where $\Omega_{s}$ is the structure domain; $\Omega_{f}$ is the fluid acoustic domain and $\Gamma_{s f}$ represents the interface between the domains.

All boundary conditions and the external force are also represented in Fig. 1. A detailed description about the formulation of the system can be found in the [21]. 


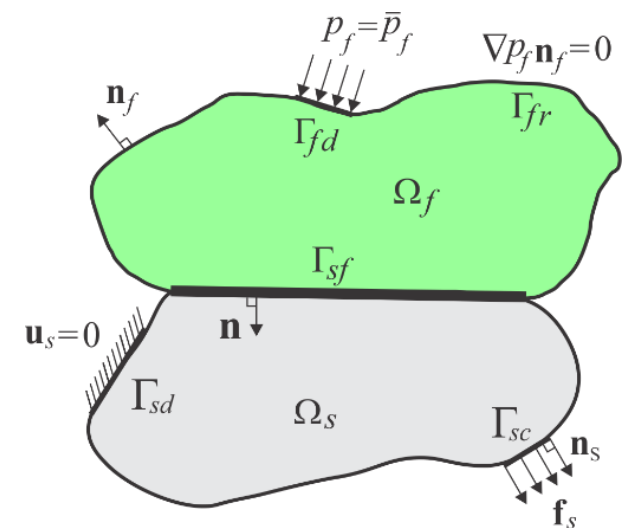

Figure 1 - Generic coupled fluid-structure system.

\subsection{Equilibrium equations and boundary conditions}

Considering the structural domain composed of a homogeneous and isotropic material and under a small linear deformation, the linear elastodynamic equation can be used to describe the continuum medium:

$$
\nabla \cdot \boldsymbol{\sigma}_{s}-\rho_{s} \frac{\partial^{2} \mathbf{u}_{s}}{\partial t^{2}}=0 \text { in } \Omega_{s}
$$

where $\nabla \cdot \boldsymbol{\sigma}_{s}$ is the divergence of the Cauchy stress tensor; $\mathbf{u}_{s}$ is the displacement vector field and $\rho_{s}$ is the mass density. The subscript $s$ denotes to structural variables.

The boundary conditions considered in the structural domain, Fig. 1, is the displacement constraint

$$
\mathbf{u}_{s}=0 \text { in } \Gamma_{s d}
$$

the prescribed external load, $\mathbf{f}_{s}$, applied in the structure

$$
\boldsymbol{\sigma}_{s} \mathbf{n}_{s}=\mathbf{f}_{s} \text { in } \Gamma_{s c}
$$

and the pressure force produced by the fluid and transmitted to the structure at the interface between the domains

$$
\boldsymbol{\sigma}_{s} \mathbf{n}_{s}=p_{f} \mathbf{n}_{f} \text { in } \Gamma_{s f}
$$

The fluid medium can be described using the wave equation, assuming the fluid to be inviscid, irrotational and in a small translation:

$$
\frac{1}{c_{0}^{2}} \frac{\partial^{2} p_{f}}{\partial t^{2}}-\nabla^{2} p_{f}=0 \text { in } \Omega_{f}
$$

where $p_{f}$ represents the acoustic pressure scalar field and $c_{0}$ is the constant speed of sound in the fluid.

In the fluid acoustic domain, the boundary conditions considered, Fig. 1, is the prescribed pressure, $\bar{p}_{f}$, in the fluid

$$
p_{f}=\bar{p}_{f} \text { in } \Gamma_{f d}
$$

the rigid wall boundary condition

$$
\nabla p_{f} \mathbf{n}_{f}=0 \text { in } \Gamma_{f r}
$$


and the kinematic compatibility of the normal displacements at the interface of the fluid and structural domains

$$
\nabla p_{f} \mathbf{n}_{f}=\rho_{f} \frac{\partial^{2} \mathbf{u}_{s}}{\partial t^{2}} \mathbf{n}_{s} \text { in } \Gamma_{s f}
$$

where $\rho_{f}$ is the mass density of the fluid medium. Them, the stated boundary value problem for acoustic-structure interaction is find $\mathbf{u}_{s}$ and $p_{f}$ that satisfy the equations [1] - [8].

\subsection{Finite element discretization}

The finite element discretization of the system is reached through taking the weak form of the boundary value problem and applying the Galerking method in the weighted residual approximation, [23]. Considering the presented coupled system of equations, the finite element matrices are written as follows

$$
\begin{gathered}
\mathbf{K}_{s}=\int_{\Omega_{s}}\left(\nabla \mathbf{N}_{s}\right)^{T} \mathbf{D}_{s} \nabla \mathbf{N}_{s} d V \\
\mathbf{M}_{s}=\rho_{s} \int_{\Omega_{s}} \mathbf{N}_{s}^{T} \mathbf{N}_{s} d V \\
\mathbf{K}_{f}=\int_{\Omega_{f}}\left(\nabla \mathbf{N}_{f}\right)^{T} \nabla \mathbf{N}_{f} d V \\
\mathbf{M}_{f}=\frac{1}{c_{0}^{2}} \int_{\Omega_{f}} \mathbf{N}_{f}^{T} \mathbf{N}_{f} d V \\
\mathbf{f}=\int_{\Gamma_{s c}} \mathbf{N}_{s}^{T} \mathbf{f}_{s} d S \\
\mathbf{L}_{s f}=\int_{\Gamma s f} \mathbf{N}_{s}^{T} \mathbf{n} \mathbf{N}_{f} d S
\end{gathered}
$$

where $\mathbf{K}_{s}$ and $\mathbf{M}_{s}$ are the stiffness and mass matrices of the structural domain; $\mathbf{K}_{f}$ and $\mathbf{M}_{f}$ are the stiffness and mass matrices of the fluid domain; $\mathbf{f}$ is the nodal vector of the external forces applied to structure; $\mathbf{L}_{s f}$ is the coupling matrix at the interface between the domains; $\mathbf{N}_{s}$ and $\mathbf{N}_{f}$ are the shape functions for the structural and fluid domains, respectively.

Letting $\omega$ be the harmonic excitation frequency of the system, the coupled fluid-structure system can be described in terms of the finite element matrices as:

$$
\left[\begin{array}{cc}
\mathbf{K}_{s}-\omega^{2} \mathbf{M}_{s} & -\mathbf{L}_{s f} \\
-\rho_{f} \mathbf{L}_{s f}^{T} \omega^{2} & \mathbf{K}_{f}-\omega^{2} \mathbf{M}_{f}
\end{array}\right]\left\{\begin{array}{l}
\mathbf{u}_{s} \\
\mathbf{p}_{f}
\end{array}\right\}=\left\{\begin{array}{l}
\mathbf{f} \\
0
\end{array}\right\}
$$

where $\mathbf{u}_{s}$ and $\mathbf{p}_{f}$ are the nodal approximation of the frequency response of displacement and pressure, respectively.

\section{EVOLUTIONARY OPTIMIZATION WITH PERIODIC CONSTRAINT}

This work aims to find the optimum layout of the periodic unit cells in the design domain in order to minimize the pressure frequency response in an acoustic-structure coupled system. Following is presented the mathematical approach of the problem and the optimization procedure used in this work. 


\subsection{Optimization problem}

A periodic system is exemplified in Fig. 2; in this generic 2D representation, the system is divided into $m=m_{1} \times m_{2}$ equal unit cells. $m_{1}$ unit cells in horizontal direction and $m_{2}$ unit cell in vertical direction, a cell mode of $m_{1} \times m_{2}$. The sensitivity number in the optimization problem is given in terms of the design variable $x_{i, j}$, where the subscript $i$ is related to cell number and the subscript $j$ refers to element number in the unit cell.

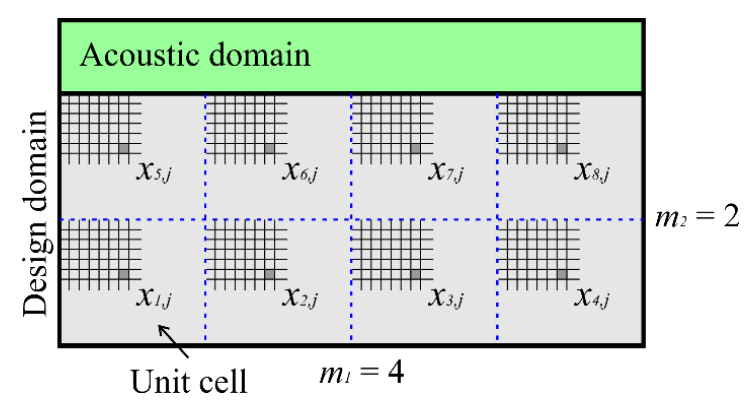

Figure 2 - Coupled system with the design domain composed of $m=8$ identical unit cells, in the cell mode $m_{1} \times m_{2}=4 \times 2$, with 4 unit cells along the horizontal direction and 2 unit cells along the vertical direction.

The optimization problem proposed here for minimizing the pressure frequency response for a periodic system can be stated as

Minimize: $\quad\left|\mathbf{p}_{f}\right|$

Subject to:

$$
\begin{aligned}
& {\left[\begin{array}{cc}
\mathbf{K}_{s}-\omega^{2} \mathbf{M}_{s} & -\mathbf{L}_{s f} \\
-\rho_{f} \mathbf{L}_{s f}^{T} \omega^{2} & \mathbf{K}_{f}-\omega^{2} \mathbf{M}_{f}
\end{array}\right]\left\{\begin{array}{l}
\mathbf{u}_{s} \\
\mathbf{p}_{f}
\end{array}\right\}=\left\{\begin{array}{l}
\mathbf{f} \\
0
\end{array}\right\}} \\
& V_{f}-m V_{i}=0 \\
& V_{i}-\sum_{j=1}^{n} v_{i, j} x_{i, j}=0 \\
& x_{1, j}=x_{2, j}=x_{3, j}=\cdots=x_{m, j} \\
& x_{i, j} \in\{0,1\} \quad(j=1,2,3, \cdots, n) \quad(i=1,2,3, \cdots, m)
\end{aligned}
$$

where $\left|\mathbf{p}_{f}\right|$ is the mean pressure frequency response in a point or area of the fluid domain; Eq. 17 refers to the equilibrium equation of the coupled system; $V_{f}$ is final prescribed volume fraction of the structural part in the design domain; $V_{i}$ the volume fraction of the $i$ th unit cell; $v_{i, j}$ is the volume fraction of the $j$ th element in the $i$ th unit cell, $n$ is the total number of elements in the unit cell and $x_{i, j}$ is the design variable with 1 for structural elements and 0 for void or fluid elements. Due the periodic constraint, the design variable is enforced to have the same value for elements with the same position in the unit cell as indicate in Eq. 20. 


\subsection{Sensitivity analysis}

Following the evolutionary optimization procedure, the sensitivity number of each element based on the objective function should be evaluated for all elements in the design domain. Then, the elements are ranked according to the they efficiency. To obtain this efficiency of each element, the equilibrium equation, Eq. 17, should be derived with respect to the design variable, $x_{i, j}$, for each element.

$$
\frac{\partial\left[\begin{array}{cc}
\mathbf{K}_{s}-\omega^{2} \mathbf{M}_{s} & -\mathbf{L}_{s f} \\
-\rho_{f} \mathbf{L}_{s f}^{T} \omega^{2} & \mathbf{K}_{f}-\omega^{2} \mathbf{M}_{f}
\end{array}\right]\left\{\begin{array}{l}
\mathbf{u}_{s} \\
\mathbf{p}_{f}
\end{array}\right\}}{\partial x_{i, j}}=\frac{\partial\left\{\begin{array}{l}
\mathbf{f} \\
0
\end{array}\right\}}{\partial x_{i, j}}
$$

Based on [22]; introducing the location vector to identify de degree of freedom for the minimization of the frequency response, neglecting the alteration in fluid global matrices due the structural element remove, the Eq. 22 can be rewritten as

$$
\frac{\partial u_{k}}{\partial x_{i, j}}=-\mathbf{u}_{s, k}^{T} \frac{\left(\mathbf{K}_{s}-\omega^{2} \mathbf{M}_{s}\right)}{x_{i, j}} \mathbf{u}_{s}
$$

where $u_{k}$ is the frequency response in the specific degree of freedom of interesting to minimize and $\mathbf{u}_{s, k}$ is frequency response of the structural domain when only a unitary load is applied in the $k$ th degree of freedom.

Appling the material interpolation proposed by [24], the sensitivity number, from Eq. 23, to minimize the proposed objective function can be state as

$$
\alpha_{i, j}= \begin{cases}\left(\mathbf{u}_{s, k}^{i, j}\right)^{T}\left(p \mathbf{K}_{s}^{i, j}-\omega^{2} \mathbf{M}_{s}^{i, j}\right) \mathbf{u}_{s}^{i, j} & x_{i, j}=1 \\ -\left(\mathbf{u}_{s, k}^{i, j}\right)^{T}\left(\omega^{2} \mathbf{M}_{s}^{i, j}\right) \mathbf{u}_{s}^{i, j} & x_{i, j}=x_{\min }\end{cases}
$$

where the superscripts $i, j$ indicate that the matrices and vector are in the elemental level. $p$ is the penalty exponent factor. Following the soft-kill procedure, the design variable is 1 for the structure elements and $x_{\min }$ for the void or fluid elements, in this work the $x_{\min }=10^{-8}$.

Due the imposed periodicity of the unit cells, the elements should be removed/added at the same time in the same position of the unit cells. In this way, the updating of the unit cell design can be done choosing a representative unit cell. And the sensitive number of the $j$ th element in the representative unit cell is calculated as the overall sensitivity number of all $j$ th elements from the other unit cells with the same position.

$$
\alpha_{j}=\sum_{i=1}^{m} \alpha_{i, j}
$$

where $\alpha_{j}$ is elemental sensitivity number of the representative unit cell, which can be choose from any unit cell once all unit cells are identical.

\subsection{Filtering and sensitivity history}

In order to prevent the instability during the topology optimization a number of strategy can be applied. A heuristic procedure that helps to avoid checkerboard patterns is filtering the elemental sensitivity number found by Eq. 25, [8]. A spatial linear filter is used here based on 
the procedure shown in [8]. Due the periodicity unit cells in the design domain the filter scheme can be applied only at the representative unit cell.

The filtering procedure starts calculating the nodal sensitivity number, $\alpha_{l}^{n}$, which is the averaging of the sensitivity number of all connected element at the node. Then, the nodal sensitivity numbers are used to calculate the elemental smoothed sensitivity numbers, $\alpha_{j}^{s}$, using a circular (for 2D, or spherical for 3D) sub-domain defined by a radius $r_{\min }$ and centered at the elements center. Finally, for each element, the nodes inside the element sub-domain have their nodal sensitivity numbers averaged into the element as follows

$$
\alpha_{j}^{s}=\frac{\sum_{l=1}^{n o d} w\left(r_{l j}\right) \alpha_{l}^{n}}{\sum_{l=1}^{n o d} w\left(r_{l j}\right)}
$$

where nod is the total number of nodes inside the sub-main; $w\left(r_{l j}\right)$ is the linear weight factor defined as

$$
w\left(r_{l j}\right)=r_{\min }-r_{l j} \quad(l=1,2, \cdots, n o d)
$$

where $r_{l j}$ is the distance between the $j$ th element centre and the node $l$.

Helping in the convergence and stability of the optimization procedure [25], a history averaging of the sensitivities is implemented and the final sensitivity elemental number, $\alpha_{j}^{f}$, for the representative cell can be calculated as follows

$$
\alpha_{j}^{f}=\frac{\left(\alpha_{j}^{s}\right)^{m}+\left(\alpha_{j}^{s}\right)^{m-1}}{2}
$$

where $m$ is the current iteration number.

\subsection{Convergence criteria}

After reached the final prescribed volume, the optimization procedure evolves until the convergence of the objective function. The prescribed error tolerance to define the convergence of the objective function is defined as [25]

$$
\text { error }=\frac{\left|\sum_{i=1}^{N}\left(\mathbf{p}_{f}\right)^{m-i+1}-\sum_{i=1}^{N}\left(\mathbf{p}_{f}\right)^{m-N-i+1}\right|}{\left|\sum_{i=1}^{N}\left(\mathbf{p}_{f}\right)^{m-i+1}\right|} \leq \tau
$$

where $\left(\mathbf{p}_{f}\right)^{m}$ is the value of the objective function in the $m$ th iteration and $N$ is the number of iterations involved in the convergence error estimation.

\section{NUMERICAL RESULTS}

The structural optimization of two acoustic-structure systems are presented in this section considering the periodicity of the design domain and applying the formulation developed in the previous sections. In the finite element discretization of the systems, the four-node quadrilateral elements are used for the both domains. 


\subsection{Example 1}

In this example, the formulation proposed in this work is applied to a coupled acousticstructure system shown in Fig. 3 for minimization of the acoustic pressure. Simulating a rotating machine, like rotary engine, turbine, electric motor, etc., a distributed harmonic load is applied to a clamped structure as presented in Fig. 3. The optimization procedure aims to minimize the acoustic pressure response in the point $P_{\text {out }}$ generated by the vibration of the structure in the frequency of $50 \mathrm{~Hz}$.

The structural domain is divided in two parts, the design domain which is considered composed by periodic unit cells and the non-design domain which is fixed and will not be modified during the optimization procedure. The non-design domain is shown in black in Fig. 3.

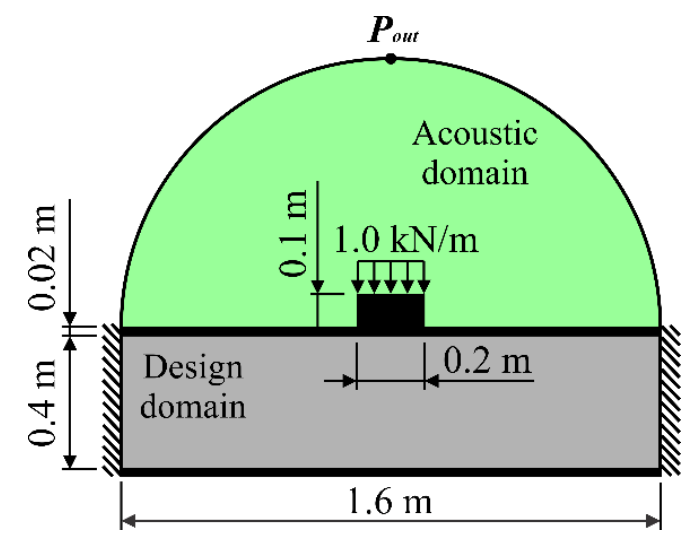

Figure 3 - Coupled system under a harmonic loading in the structure domain for minimization of the acoustic pressure at the top of the acoustic domain.

The follow material properties are adopted for the structural domain: Young's modulus $E=200 \mathrm{GPa}$, Poisson's ratio $\mu=0.3$ and density $\rho=7700 \mathrm{~kg} / \mathrm{m}^{3}$. For the acoustic fluid domain, the air properties are considered: density $\rho=1.2 \mathrm{~kg} / \mathrm{m}^{3}$ and the speed of sound $c=343 \mathrm{~m} / \mathrm{s}$. The volume of the structural domain is kept constant during the optimization procedure. Four different cell modes configuration of the initial periodic design are optimized, $4 \times 1,8 \times 2,12 \times 3$ and $16 \times 4$. For a fair comparison of the unit cell final topology, the unit cell for all configuration has a mesh of $140 \times 140$ elements.

The BESO parameters [8] used are: $E R=0.02, A r=0.02, \tau=0.005, N=5$ and $p=3$. For all cell mode configurations, the filter radius is defined as $1 / 10$ of the side length of the unit cell. The initial and final volume fraction of the design domain is defined as $V_{f}=V_{i}=0.55$.

Figure 4 shows the pressure distribution for the initial configurations of the system and for the optimized design domain considering the frequency excitation of $50 \mathrm{~Hz}$. The sensitivity number distribution in the optimized unit cell is also shown in Fig. 4 for all configurations of the periodic structure. The unit cell final topology converges for a symmetric pattern as the number of unit cell increases in horizontal and vertical directions. For the cell modes $12 \times 3$ and $16 \times 4$ the optimized unit cells show only a slight difference in the distribution of the material and can be considered as the converged pattern. 


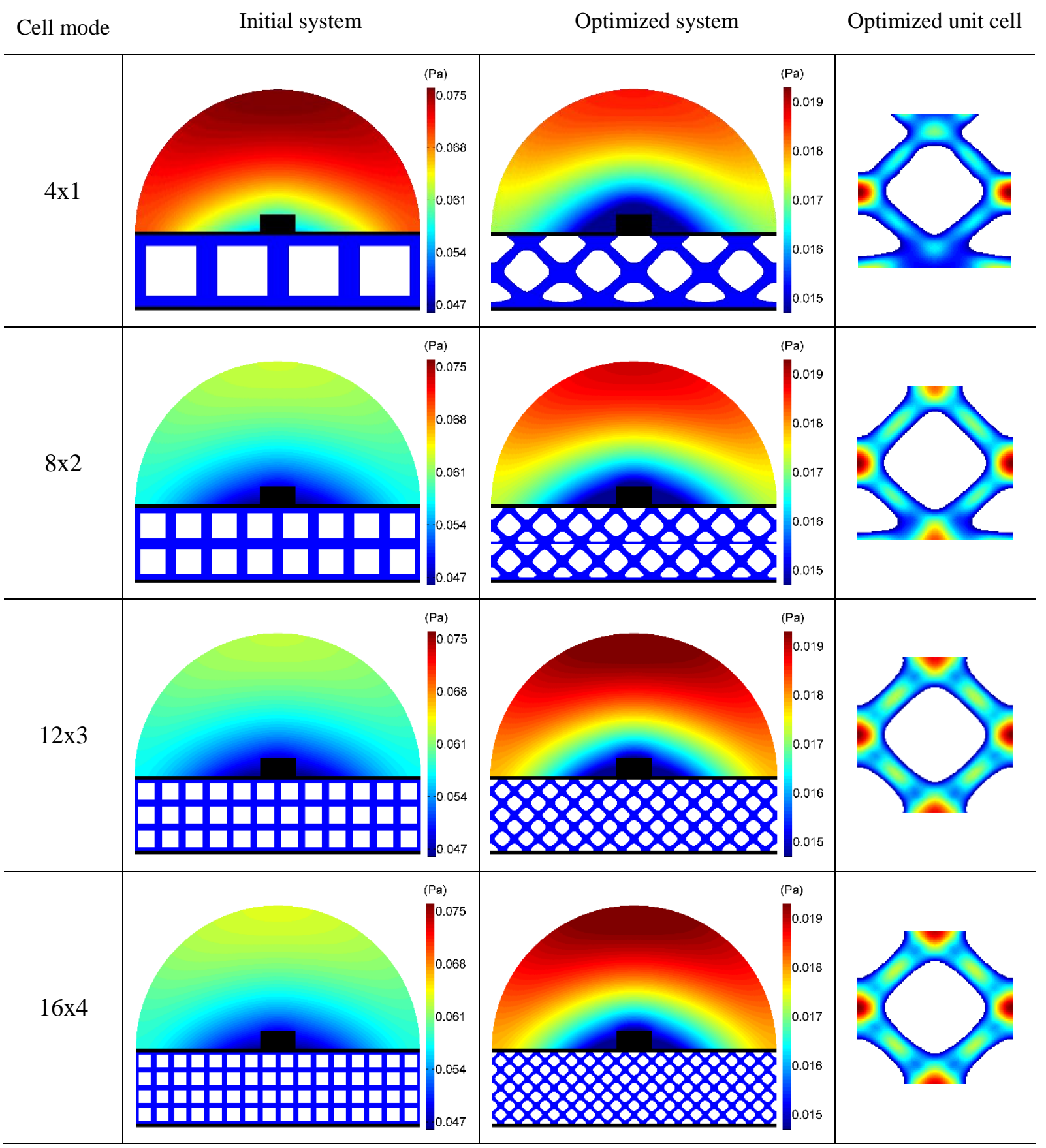

Figure 4 - Pressure distribution for the initial guess design and for the optimized configurations considering four different cell modes: $4 \times 1,8 \times 2,12 \times 3$ and $16 \times 4$. Evolution of the optimized unit cell for different cell modes.

The history of the objective function during the evolutionary optimization can be seen in Fig. 5 for all cases analyzed. The volume of the unit cell is kept constant for all cases during the optimization. Smooth convergences are observed for all configurations studied, the objective function curves do not present any peaks or discontinuities, the small value of the parameter $A r$ assure that only a small percentage of the structural material will be swapped in each iteration on the unit cells. The maximum number of iteration needed to satisfied to convergence criteria was 34 in the first case. 


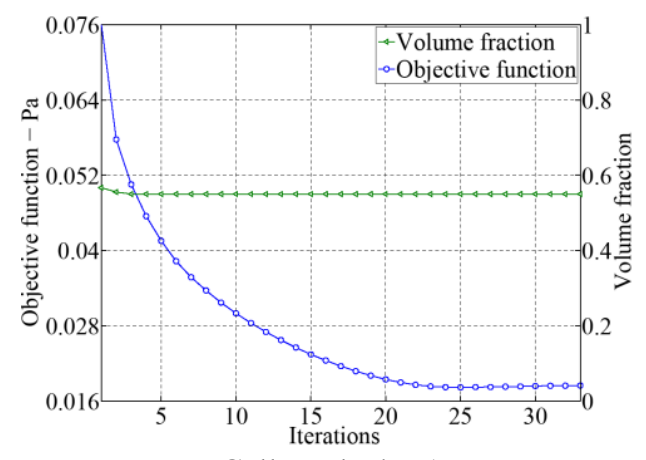

Cell mode 4 x 1

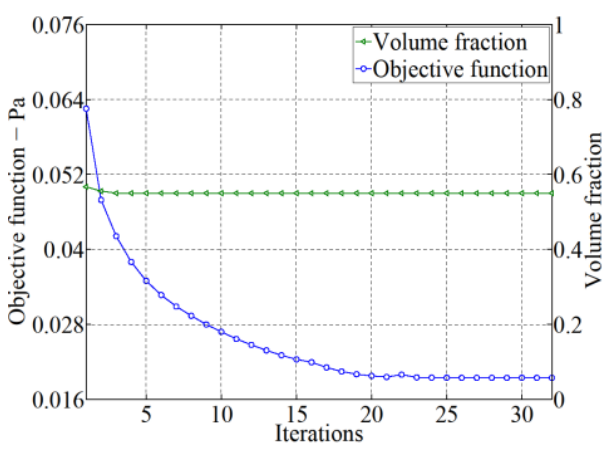

Cell mode 12 × 3

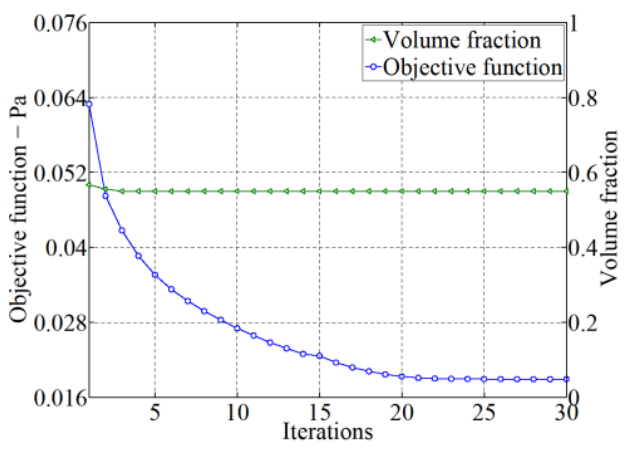

Cell mode $8 \times 2$

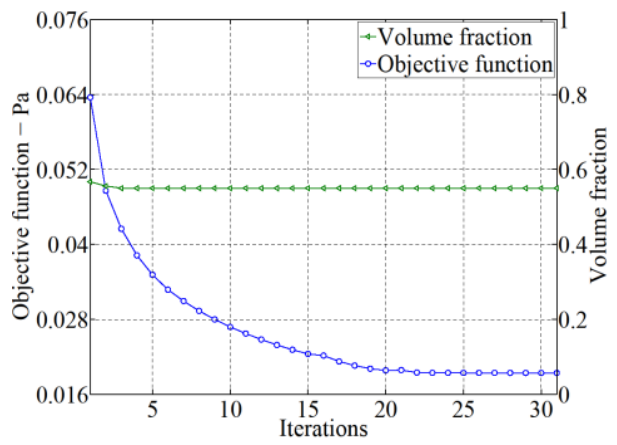

Cell mode 16 x 4

Figure 5 - Evolutionary histories of the objective function, the acoustic pressure in the point $P_{\text {out }}$, for the four different cell modes analyzed.

A new periodic unit cell is considered to be studied in the same coupled system for minimizing the acoustic pressure. The design domain is considered to be composed of periodic unit cell with the ratio length/high equal to 2 . The same materials properties and the BESO parameters for the previous analyses are used. Figure 6 shows initials and optimized topologies of the periodic system with the new unit cell, 4 cell modes are presented $2 \times 1,4 \times 2,6 \times$ 3 and $8 \times 4$. For all cases the mesh of the unit cell is discretized with $200 \times 100$ elements.

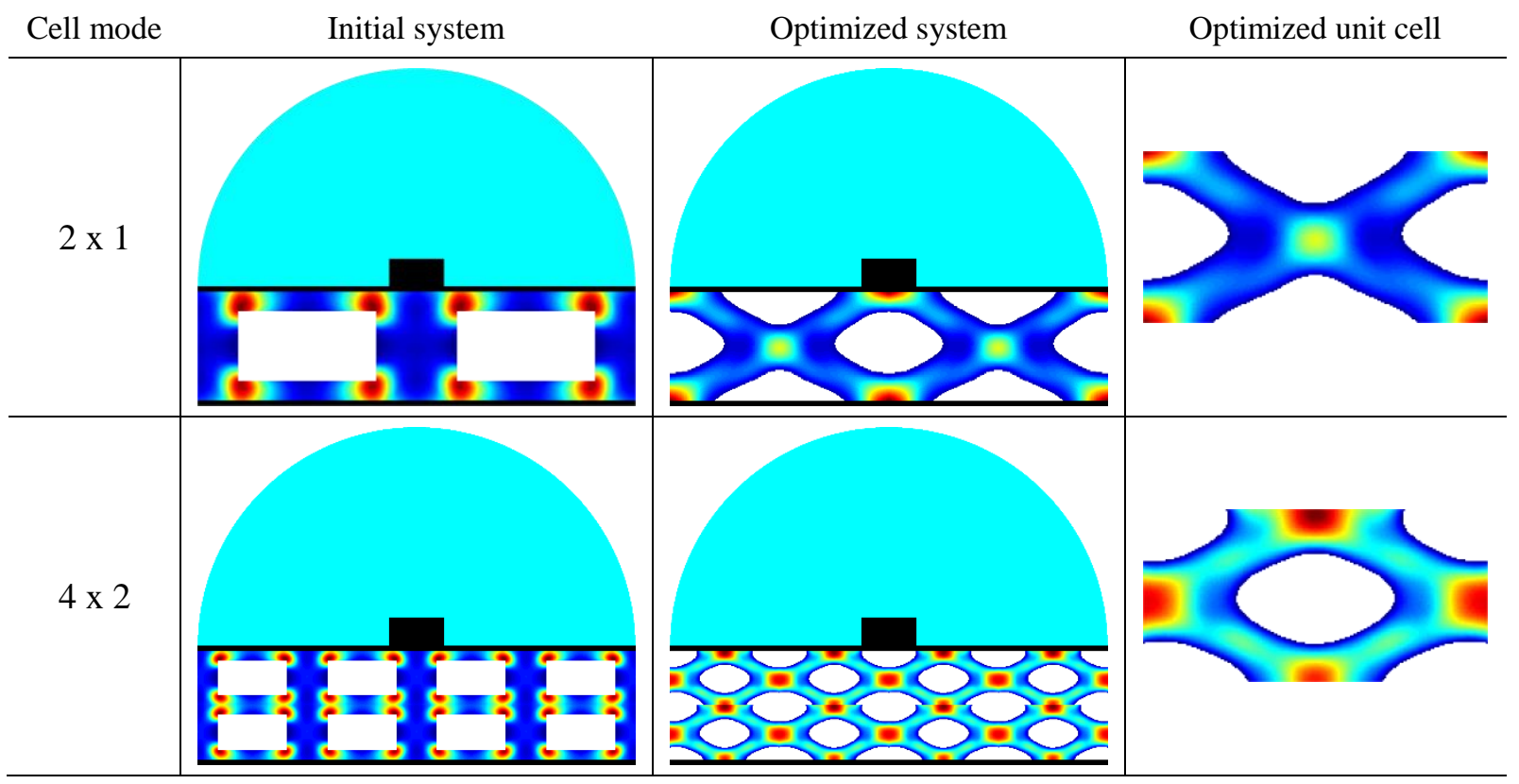




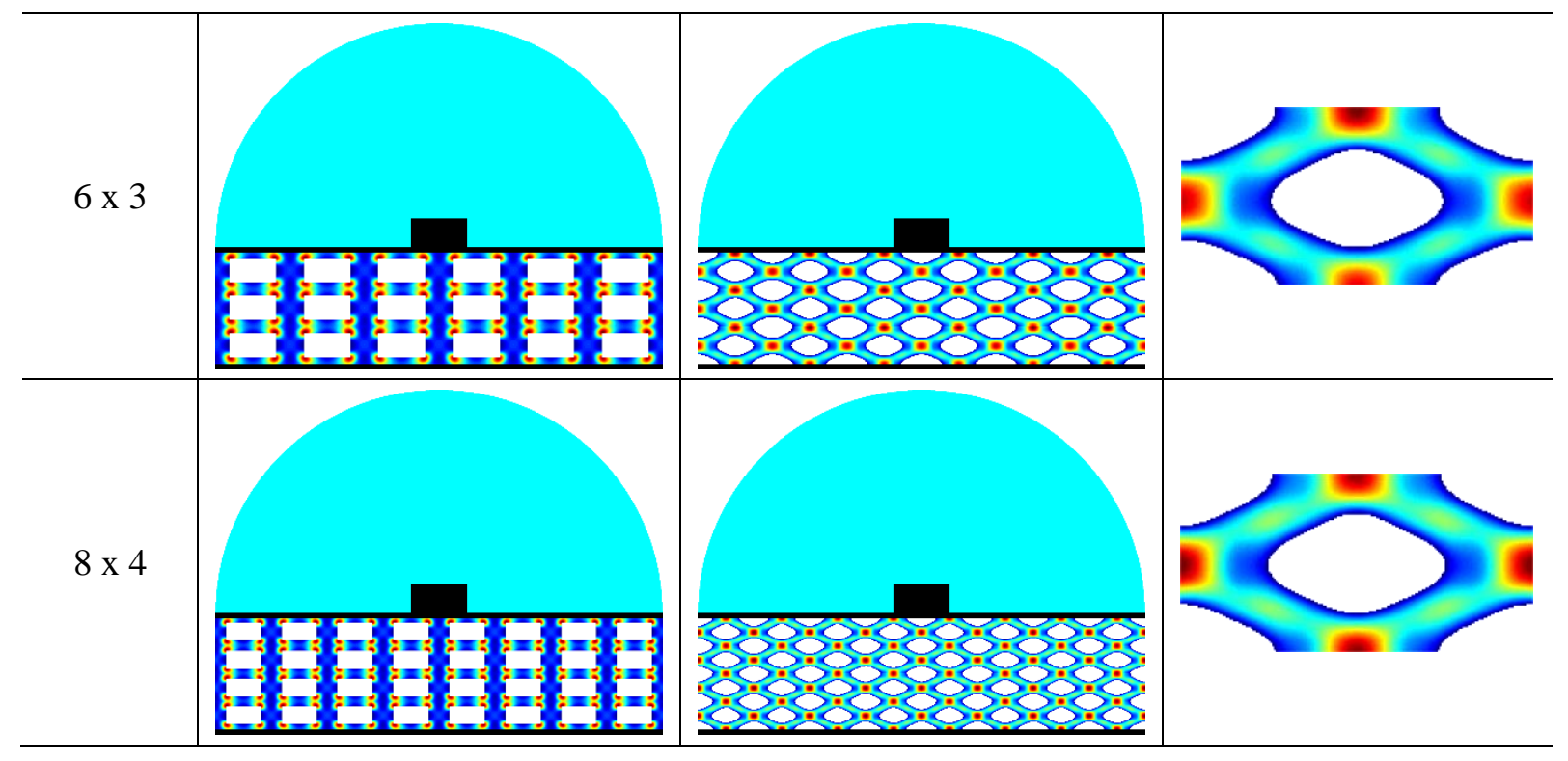

Figure 6 - Initial guess design and optimized final topologies of the coupled system considering four different cell modes: $2 \times 1,4 \times 2,6 \times 3$ and $8 \times 4$. Optimized unit cell for different configuration of the design domain.

The convergence of the unit cell to pattern is observed for the cell mode $6 \times 3$ and $8 \times 4$ and is expected to be repeated in cell modes with higher number of cells. Figure 7 presents the history of the objective function in the optimization procedure for all cell modes investigated. The volume of the unit cell is kept constant during the optimization, $V_{f}=V_{i}=0.65$.

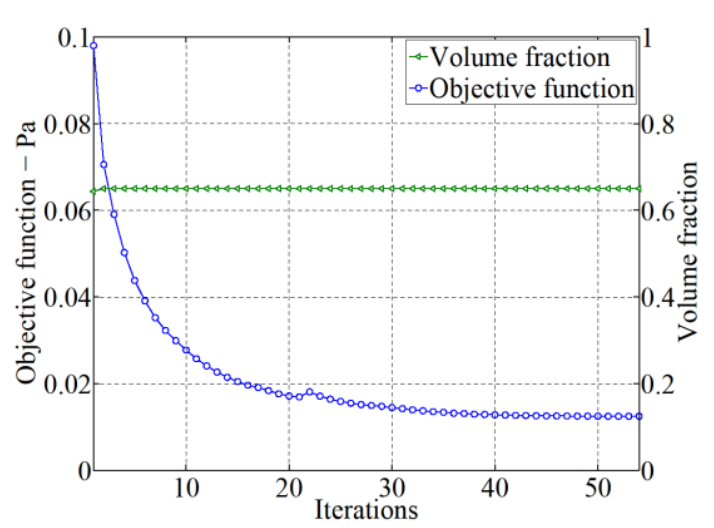

Cell mode 2 x 1

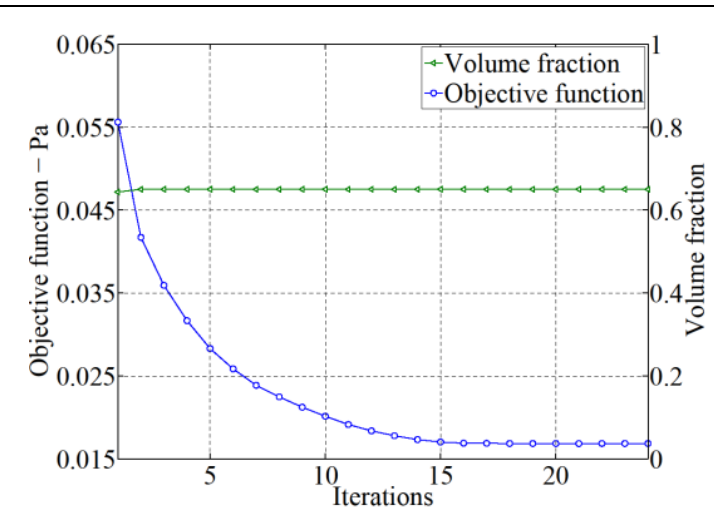

Cell mode $6 \times 3$

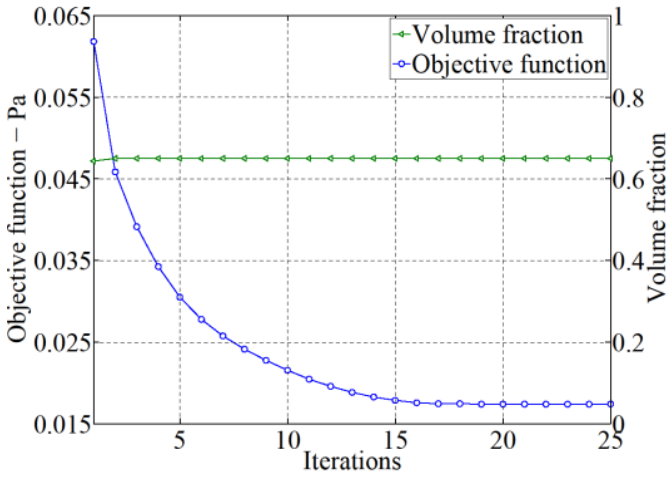

Cell mode 4 x 2

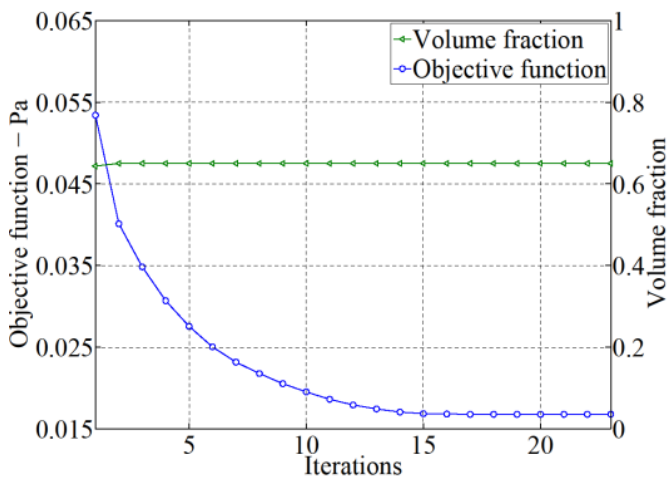

Cell mode 8 x 4

Figure 7 - Evolutionary histories of the objective function for the different cell modes analyzed with the new unit cell aspect ratio. 
Can be observed in Fig. 7, that the number of iteration needed to reach the convergence criteria is decreasing with the increase of the number of periodic cells.

The topology optimization of system is now explored for a higher frequency of excitation. Instead of the frequency of $50 \mathrm{~Hz}$ previous analyzed, now a frequency of $350 \mathrm{~Hz}$ is taking into account to the optimization of the periodic design domain of the coupled system showed in Fig. 3. The cell mode of 32 unit cells $(4 \times 8)$ is used to build the design domain. The same previous material properties and BESO parameters are applied in this investigation. The initial guess design of the unit cell start with a structural volume fraction of 0.5 , however, the void area in the unit cell is fill with acoustic fluid. In this way, the couple system presents now only two phases, acoustic fluid or structure material in all stages of the optimization procedure.

Figure 8 presents the initial and final topologies of the coupled system considering the optimization for the frequency of $350 \mathrm{~Hz}$. The pressure distributions in the acoustic domain and in the design domain for both initial and final topologies. The vibration operational modes are presented considering the initial and the optimized system.

Initial system

Optimized system

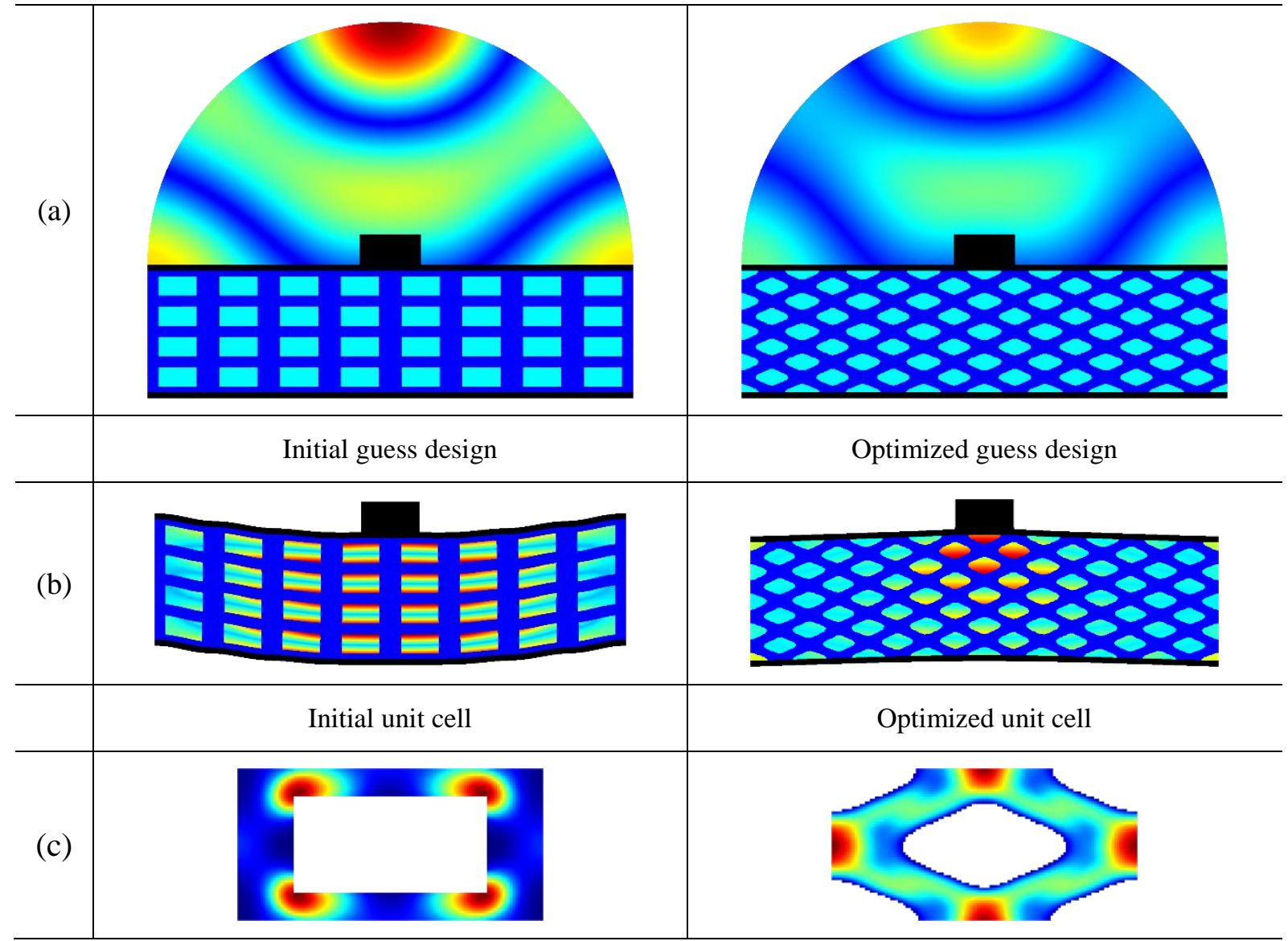

Figure 8 - Topology optimization of the couple acoustic-structure system considering the frequency of $350 \mathrm{~Hz}$ : (a) Pressure distribution on the acoustic domain for the initial and the optimized system; (b) Frequency response of displacement and pressure distributions in the design domain; (c) Sensitivity number distribution in the initial guess design and in the optimized unit cell.

The system frequency response, mean acoustic pressure, in the point $P_{\text {out }}$ is show in Fig. 9 for initial system and for the optimized system. The response of the system indicates a significant reduction of the acoustic pressure in the point $P_{\text {out }}$ for the frequency range of $(0-380) \mathrm{Hz}$. 


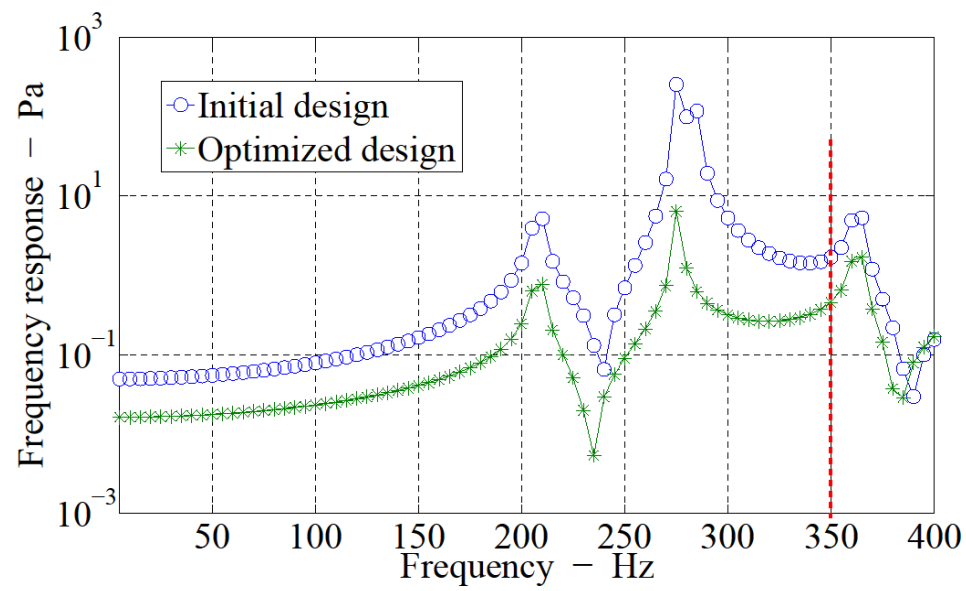

Figure 9 - Frequency response in the $P_{\text {out }}$ in the acoustic domain considering the initial and the optimized design of the coupled system.

\subsection{Example 2}

The optimization of a coupled acoustic-structure system composed of two acoustic cavities separated by a structural domain is investigated in this example. As shown in Fig. 10, a harmonic pressure is imposed in the first acoustic cavity, $P_{i n}$, and transmitted to the second cavity by the vibration of the clamped structure. The optimization procedure aims to minimize the pressure in the second cavity in the point $P_{\text {out }}$. The boundary conditions and geometric dimensions of the system are also shown in Fig. 10. The excitation frequency of the system is $f=50 \mathrm{~Hz}$. The whole structural part is considered to be the design domain and its composed of periodic unit cells. A number of cell modes is investigated in order to obtain a convergent pattern of the unit cell. In all configurations, each unit cell is squared and discretized using $200 \times 200$ elements.

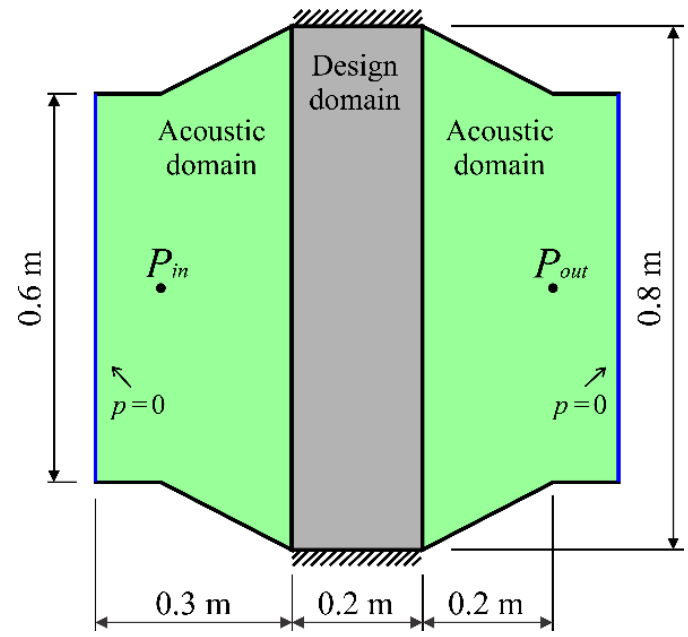

Figure 10 - Acoustic-structure system with a harmonic imposed pressure, $P_{i n}$, for minimize of the acoustic pressure at specific point, $P_{\text {out }}$, in the other cavity.

The acoustic fluid has the follow properties: density $\rho=1.2 \mathrm{~kg} / \mathrm{m}^{3}$ and the speed of sound $c=343 \mathrm{~m} / \mathrm{s}$. The design domain material has Young's modulus $E=100 \mathrm{MPa}$, Poisson's ratio $\mu=0.3$ and density $\rho=100 \mathrm{~kg} / \mathrm{m}^{3}$. 
The BESO parameters used are: $E R=0.02, A r=0.02, \tau=0.01, N=5$ and $p=3$. For all cell mode configurations, the filter radius is defined as $6 \%$ of the side length of the unit cell. The optimization is performed with a constant volume fraction of the design domain $V_{f}=V_{i}=0.40$.

Three different cell modes are investigated in order to obtain the convergence of the unit cell. Figure 11 presents the pressure distribution in the acoustic cavities and inside unit cells in the design domain for cases analyzed, cell modes $1 \times 4,2 \times 8$ and $3 \times 12$.

The optimized unit cells for the cell modes $2 \times 8$ and $3 \times 12$ are similar with one a slender variation of the topology.

The sensitivity number distribution in the optimized unit cell is also shown in Fig. 11 for all configurations of the periodic structure. The unit cell final topology converges for a symmetric pattern as the number of unit cell increases in horizontal and vertical directions. For the cell modes $2 \times 8$ and $3 \times 12$ the optimized unit cells show only a slender difference in the distribution of the material and can be considered as the converged pattern.

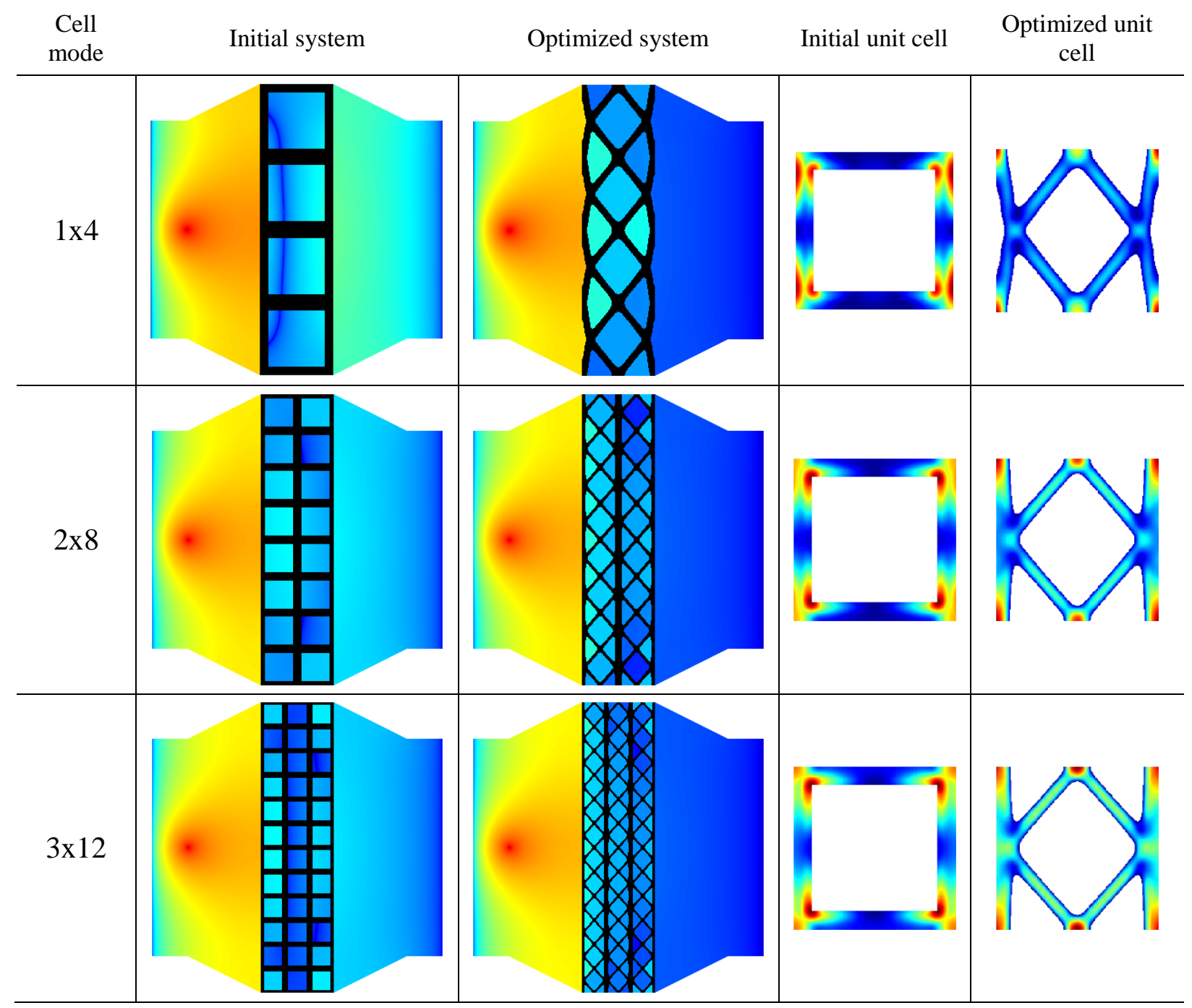

Figure 11 - Initial guess design and optimized final topologies of the fluid-structure system considering different cell modes: 1 x 4, 2 × 8 and $3 \times 12$. Initial and optimized unit cell for three cell modes different configurations.

Evolutionary history of the topology for the unit cell optimization is presented in Fig. 12 considering the first cell mode configuration 1 x 4 . 

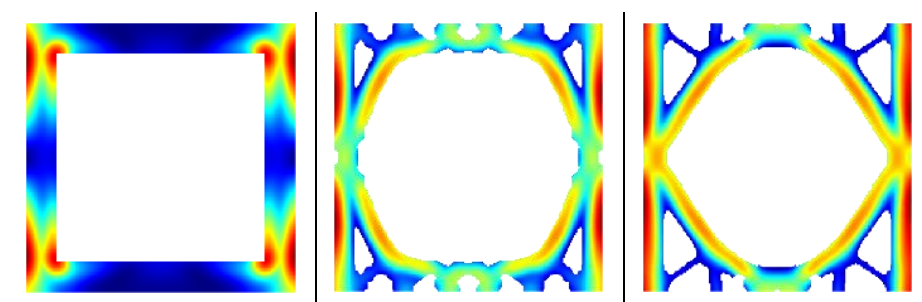

Initial guess design

Iteration 10

Iteration 20

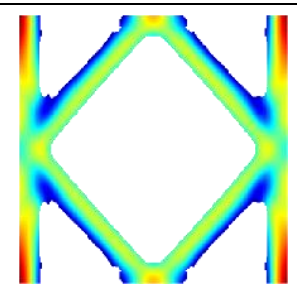

Iteration 30

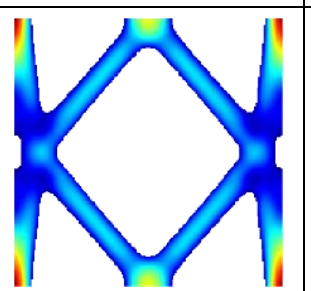

Iteration 40

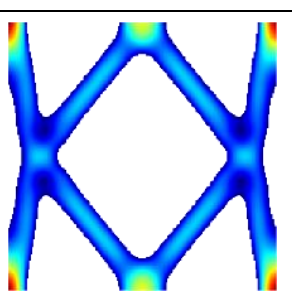

Iteration 58

Figure 12 - Sensitivity number distribution on the evolutionary history of topology for the cell mode 1 x 4 case.

Smoothed convergences of the objective function are observed in Fig. 13 for all cell modes. The cell mode configuration $1 \times 4$ needed the highest number of iteration to satisfied the convergent criteria, 58 iterations. The initial and final objective function for the cell modes $1 \times 4$, $2 \times 8$ and $3 \times 12$ are $(0.787-0.014) \cdot 10^{-2} \mathrm{~Pa},(0.211-0.016) \cdot 10^{-2} \mathrm{~Pa}$ and $(0.170-0.016) \cdot 10^{-2}$ $\mathrm{Pa}$, respectively. The cell mode $1 \times 4$ shows the best performance in this case. The configuration with more number of periodic unit cells, $3 \times 12$, required the lowest number of iterations in order to converge, 45 iterations.

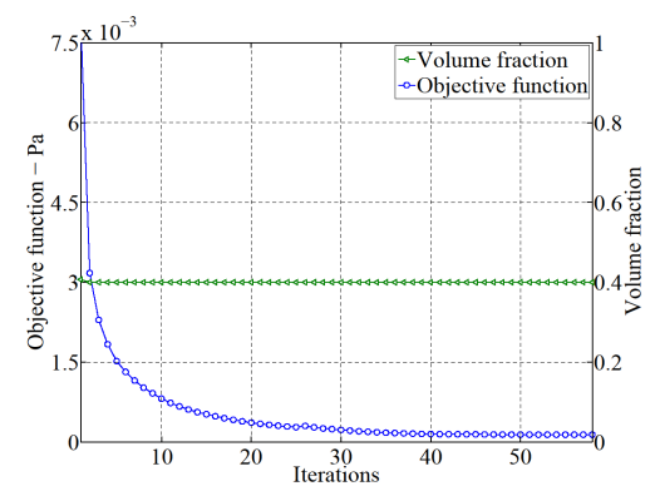

Cell mode 1 x 4

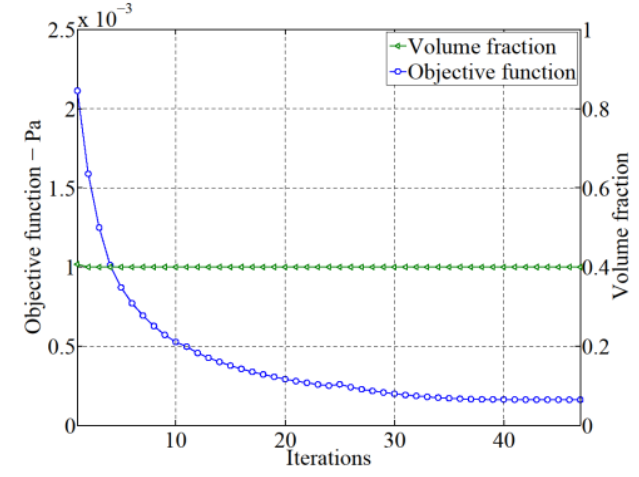

Cell mode 2 × 8

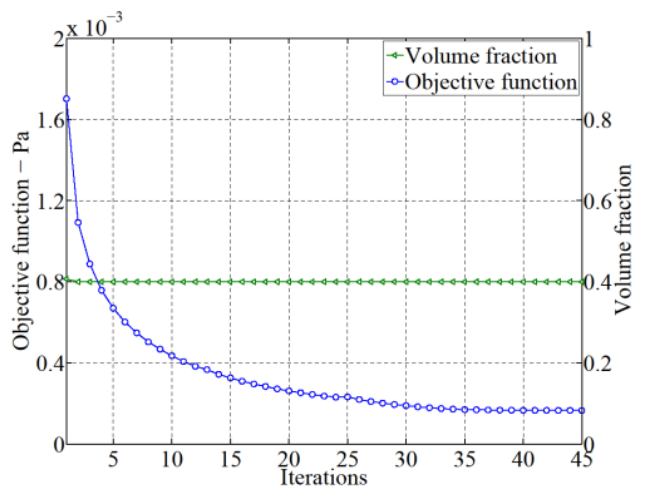

Cell mode $3 \times 12$

Figure 13 - Evolutionary histories of the objective function for all three cell modes of the design domain. The constant structural volume fraction of 0.4 for all cases during the optimization procedure. 
The frequency response of the objective function, the mean acoustic pressure at point $P_{\text {out }}$, is presented in Fig. 14 considering the initial and the optimized coupled system. The frequency of interesting in the optimization procedure, $50 \mathrm{~Hz}$, is indicated with the red line. Can be seen that the first natural frequency of the system increased from 60 to $185 \mathrm{~Hz}$, approximately, as a consequence of the optimization process. The objective function decreased not only in the specific frequency, but in the range of $(0-140) \mathrm{Hz}$.

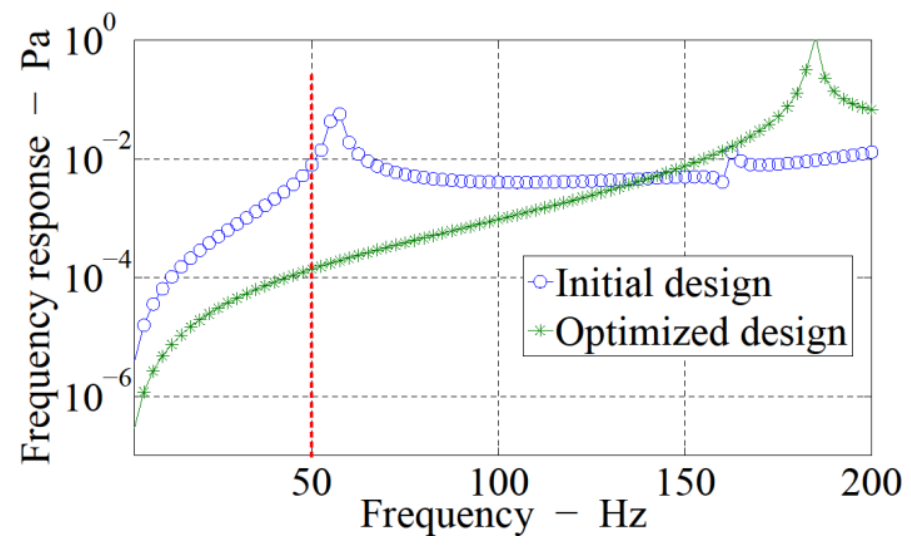

Figure 14 - Frequency response considering the acoustic pressure in the point $P_{\text {out }}$ for the initial and the optimized design of the coupled acoustic-structure system.

\section{CONCLUSIONS}

A topology optimization methodology of periodic acoustic-structure systems has been presented in this work. The pressure frequency response is defined as the objective function in the optimization formulation. Two examples exploring different aspects of the proposed methodology are presented. The following conclusions are drawn:

- The proposed optimization procedure can effectively minimize the pressure frequency response in the acoustic domain of coupled acoustic-structure systems.

- A periodic geometry constraint has been implemented for the optimization of the coupled systems to obtain periodic unit cells in the design domain.

- Considering the unit cell composed of structural or void areas or composed of fluid and structural material, the minimization of the objective function and the convergence of the unit cell to a pattern have been reached in all analyzed examples.

- Analyzing systems with several different cell modes, the value of the objective function and the optimal topology tend to converge as the total number of periodic unit cell grows. In the cases studied, at least three unit cells in each direction is need to obtain the convergence of the unit cell topology.

- The minimum values of the objective function in the analyzed cases have been found for the configurations with the lowest numbers of unit cells. Nevertheless, the configurations with higher numbers of unit cells could be more versatile and have lower cost to construct using additive manufacturing once the unit cell topology have converged to a pattern. 


\section{ACKNOWLEDGMENTS}

The authors are grateful to FAPESP (São Paulo Research Foundation, grant numbers 2011/09730-6, 2013/08293-7 and 2013/00085-6) for the financial support of this work.

\section{REFERENCES}

[1] M. P. Bendsoe and N. Kikuchi, "Generating optimal topologies in structural design using a homogenization method," Comput. Methods in Appl. Mech. Eng., vol. 71, pp. 197-224, 1988.

[2] M. Zhou and G. I. N. Rozvany, "The COC algorithm, part II: topological, geometrical and generalized shape optimization," Comput. Methods in Appl. Mech. Eng., vol. 89, pp. 309-336, 1991.

[3] M. P. Bendsoe and O. Sigmund, Topology Optimization - Theory, Methods and Applications, Berlin: Springer Verlag, 2003, p. 370.

[4] Y. M. Xie and G. P. Steven, "A simple evolutionary procedure for structural optimization," Comput. Struct., vol. 49, pp. 885-896, 1993.

[5] Y. M. Xie and G. P. Steven, Evolutionary Structural Optimization, Springer, London, 1997.

[6] J. Sethian and A. Wiegmann, "Structural Boundary Design via Level Set and Immersed Interface Methods," J. Comput. Phys., vol. 163, no. 2, pp. 489-528, 2000.

[7] M. Y. Wang, X. Wang and D. Guo, "A level set method for structural topology optimization," Comput. Methods in Appl. Mech. Eng., vol. 192, no. 1-2, pp. 227-246, January 2003.

[8] X. Huang and Y. M. Xie, Evolutionary Topology Optimization of Continuum Structures: Methods and Applications, Chichester: John Wiley \& Sons, Ltd, 2010, p. IX+223.

[9] G. H. Yoon, J. S. Jensen and O. Sigmund, "Topology optimization of acoustic-structure problems using a mixed finite element formulation," Int. J. Numer. Meth. Eng., vol. 70, pp. 1049-1075, 2007.

[10] L. Shu, M. Y. Wang and Z. Ma, "Level set based topology optimization of vibrating structures for coupled acoustic-structural dynamics," Comput. Struct., vol. 132, pp. 3442, 2014.

[11] J. Du and N. Olhoff, "Minimization of sound radiation from vibrating bi-material structures using topology optimization," Struct. Multidisc. Optim., vol. 33, pp. 305-321, 2007.

[12] J. Du and N. Olhoff, "Topological design of vibrating structures with respect to optimum sound pressure characteristics in a surrounding acoustic medium," Struct. Multidisc. Optim., vol. 42, pp. 43-54, 2010.

[13] W. M. Vicente, R. Picelli and R. Pavanello, "An Evolutionary Structural Optimization applied to Fluid-Structure Problems," in Proceedings of the Eleventh International Conference on Computational Structures Technology, CST 2012, Dubrovnik, Croatia., 2012.

[14] R. Picelli, W. M. Vicente and R. Pavanello, "Bi-directional evolutionary structural optimization for design-dependent fluid pressure loading problems," Eng. Optimiz., vol. 47, no. 10, pp. 1324-1342, 2015. 
[15] R. Picelli, W. M. Vicente, R. Pavanello and Y. M. Xie, "Evolutionary topology optimization for natural frequency maximization problems considering acoustic-structure interaction," Finite Elem. Anal. Des., vol. 106, pp. 56-64, 2015.

[16] O. Sigmund, "Materials with prescribed constitutive parameters: An inverse homogenization problem," Int. J. Solids. Struct., vol. 31, no. 17, pp. 2313-2329, 1994.

[17] O. Sigmund and S. Torquato, "Design of materials with extreme thermal expansion using a three-phase topology optimization method," J. Mech. Phys. Solids, vol. 45, no. 6, pp. 1037-1067, 1997.

[18] X. Huang and Y. M. Xie, "Optimal design of periodic structures using evolutionary topology optimization," Struct. Multidisc. Optim., vol. 36, no. 6, pp. 597-606, 2007.

[19] Z. H. Zuo, X. Huang, X. Yang, J. H. Rong and Y. M. Xie, "Comparing optimal material microstructures with optimal periodic structures," Comp. Mater. Sci., vol. 69, pp. 137147, 2013.

[20] Z. H. Zuo, Y. M. Xie and X. Huang, "An improved bi-directional evolutionary topology optimization method for frequencies," Int. J. Struct. Stab. Dy., vol. 10, pp. 55-75, 2010.

[21] W. M. Vicente, R. Picelli, R. Pavanello and Y. M. Xie, "Topology optimization of frequency responses of fluid-structure interaction systems," Finite Elem. Anal. Des., vol. 98, pp. 1-13, 2015.

[22] W. M. Vicente, Z. H. Zuo, R. Pavanello, T. K. L. Calixto, R. Picelli and Y. M. Xie, "Concurrent topology optimization for minimizing frequency responses of two-level hierarchical structures," Comput. Methods in Appl. Mech. Eng., vol. 301, pp. 116-136, 2016.

[23] K. J. Bathe, Finite Element Procedures in Engineering Analysis, Englewood Cliffs, NJ: Prentice-Hall, 1982.

[24] X. Huang, Z. H. Zuo and Y. M. Xie, "Evolutionary topological optimization of vibrating continuum structures for natural frequencies," Comput. Struct., vol. 88, pp. 357-364, 2010.

[25] X. Huang and Y. M. Xie, "Convergent and mesh-independent solutions for the bidirectional evolutionary structural optimization method," Finite Elem. Anal. Des., vol. 43, pp. 1039-1049, 2007. 